\title{
Prevention of Sudden Cardiac Death in Dialysis Patients: Drugs, Defibrillators or What Else?
}

\author{
Rod Passman \\ Northwestern University Feinberg School of Medicine, Chicago, III., USA
}

\section{Key Words}

Cardiomyopathy • Dialysis-related risk factors •

Electrophysiologic instability $\cdot$ Ischemic heart disease •

Sudden cardiac death

\begin{abstract}
Death from cardiovascular disease in general and sudden cardiac arrest (SCA) in particular are exponentially proportional to declining renal function and are a major cause of mortality among those with chronic kidney disease (CKD). The greatest risk, however, is reserved for those patients on chronic dialysis. These individuals have an extraordinarily high rate of death, with cardiac disease accounting for $45 \%$ and SCA responsible for $25 \%$ of all-cause mortality. Once cardiac arrest occurs, survival is extremely poor. Thus, reducing mortality from cardiovascular disease and SCA in dialysis patients is a global health challenge. The main objectives of this review are to elucidate the nature of SCA in the dialysis population, describe possible mechanisms and risk factors, and discuss options for prevention.
\end{abstract}

Copyright $\odot 2013$ S. Karger AG, Basel

\section{Introduction}

Cardiovascular mortality is inversely and exponentially proportional to renal function and is a major cause of death among all patients with chronic kidney disease
(CKD). The presence of even mild renal insufficiency is associated with a $40 \%$ increase in cardiac death and a $300 \%$ increase in the risk of sudden cardiac arrest (SCA) compared to those with normal renal function [1]. The greatest risk of cardiovascular and sudden death, however, is reserved for those patients on chronic dialysis. These patients have an extraordinarily high mortality, with cardiovascular disease and SCA accounting for 60 and $25 \%$ of all-cause mortality, respectively [2]. Once cardiac arrest occurs in this group of patients, survival is poor even if it occurs in the controlled setting of a dialysis unit. With 600,000 individuals currently receiving renal replacement therapy in the United States alone, reducing mortality from cardiovascular disease and SCA among dialysis patients is a global health challenge.

\section{Definitions of Sudden Cardiac Death: Do They Apply to the CKD Patient?}

Traditional definitions of SCA include either a witnessed cardiac arrest which occurs suddenly and within an hour of symptom onset or an unwitnessed death which is unexpected in a patient known to be well in the last $24 \mathrm{~h}$ without a clear non-cardiac cause of death. Whether these definitions apply to dialysis patients who spend a disproportionate amount of time in a healthcare setting and have significant competing risks of death from other causes is a matter of debate.

\section{KARGER}

Fax +41613061234

E-Mail karger@karger.ch

www.karger.com
(C) 2013 S. Karger AG, Basel

0253-5068/13/0353-0049\$38.00/0

Accessible online at:

www.karger.com/bpu
Rod Passman, MD, MSCE

Northwestern Cardiology Division

676 North St. Claire, Suite 600

Chicago, IL 60611 (USA)

E-Mail r-passman@northwestern.edu 




Fig. 1. Potential causal factors of SCA (adapted from Herzog et al. [5]).

In patients without CKD, the proportion of SCA due to ventricular tachycardia (VT) and ventricular fibrillation (VF) is estimated at $50-75 \%$. Whether VT/VF is equally represented in patients with mild to moderate CKD is unknown, but there is evidence to suggest substantial variance in the dialysis population. In a case series of cardiac arrest calls in a single dialysis unit, VT/VF was found in only $16 \%$ at initial evaluation [3]. An autopsy series of chronic dialysis patients revealed that the majority of deaths thought to be due to SCA were actually due to vascular events (i.e. stroke, ruptured aneurysms) and not arrhythmias [4]. These findings raise the possibility that the risk of arrhythmic death in dialysis patients may be overestimated in some settings.

\section{Mechanisms and Risk Factors}

Sudden death occurs from a confluence of arrhythmogenic triggers superimposed on a vulnerable substrate. For chronic dialysis patients, both vulnerable substrate and triggers exist in abundance. Underlying structural abnormalities including left ventricular hypertrophy (LVH), systolic dysfunction, coronary artery disease (CAD), and arteriosclerotic vascular disease provide just some of the anatomic milieu for malignant arrhythmias. Fluid and electrolyte shifts, divalent ion abnormalities, derangements in autonomic function, and abnormalities in cardiac conduction represent just some of the potential triggers (fig. 1) [5].

\section{Cardiac Risk Factors}

\section{Ischemic Heart Disease}

The prevalence of atherosclerotic heart disease increases as renal function declines. Renal dysfunction predisposes the patients to not only the risk of developing atherosclerosis but also acts synergistically to increase the complications of the disease process. Coronary lesions in CKD are characterized by a distinct intima-media thickening and calcification of the coronary arteries. Hyperphosphatemia and associated secondary hyperparathyroidism result in non-compliant vessels due to vascular calcification with smooth muscle proliferation. Rates of myocardial infarction and survival after myocardial infarction are adversely affected by CKD [6].

Though obstructive CAD is an important contributor to SCA in dialysis patients, it is clearly not the only one. Clinical trial data reveal that direct CAD-related deaths account for only $9 \%$ of all-cause mortality whereas SCA accounts for one-quarter [7]. An analysis of data from the USRDS found a 2-year mortality of 48 and $43 \%$, respectively, after non-drug eluting coronary stents and coronary artery bypass surgery in the dialysis population with annual mortality attributed to arrhythmic mechanisms estimated at approximately $8 \%$. This data implies that while revascularization may have some importance in preventing SCA, the sole reliance on this intervention may be an inadequate strategy in dialysis patients [8].

\section{Cardiomyopathy}

Congestive heart failure is present in one-third of patients at the initiation of dialysis. Three forms of uremic cardiomyopathy are described and include: (1) LVH, (2) dilation, and (3) systolic dysfunction. Left ventricular dysfunction and LVH are far more common in dialysis patients compared to the general population. Ejection fractions below 35\% are seen in approximately 1 in 7 dialysis patients and LVH is present in as much as $80 \%$ [9]. Interstitial fibrosis and endothelial dysfunction represent the main pathophysiological mechanisms underlying these cardiomyopathies. Arterial hypertension is documented in the majority of patients and is a well-established major risk factor for LVH. Anemia, fluid overload, 
and the presence of an arteriovenous fistula result in volume overload which also promotes the development of left ventricular dilation and hypertrophy. These structural abnormalities may ultimately lead to diastolic and systolic dysfunction and their attending risks of heart failure and sudden death. MRI evaluation of hemodialysis patients suggests that hypertrophy is the predominant form of uremic cardiomyopathy with left ventricular dilation and systolic dysfunction more the result of infarction or diffuse fibrosis [10]. The presence of LVH in the dialysis cohort carries important prognostic value with a left ventricular mass index of $>125 \mathrm{~g} / \mathrm{m}^{2}$ associated with a $30 \%$ increase in mortality. Whether or not this is due to sudden death is uncertain, but a higher rate of arrhythmias can be anticipated due to ischemia, the presence of myocardial scar, and the prolongation in ventricular repolarization associated with increases in left ventricular mass [11].

In all individuals, systolic dysfunction correlates with cardiac survival, but the threshold for increasing risk may be different in patients on dialysis. In the non-dialysis population, an ejection fraction of less than $35-40 \%$, regardless of etiology, is the most potent risk factor for SCA. This is not clearly the case in dialysis patients where the majority of SCA occurs in those with ejection fractions $>35 \%$ [12]. This also appears to hold true for peritoneal dialysis patients where an ejection fraction of $\leq 48 \%$ was found to be an independent predictor of SCA, emphasizing that even mild degrees of systolic dysfunction may be all that is necessary to precipitate events in the setting of renal replacement therapy [13].

\section{Electrophysiologic Instability}

The propensity for life-threatening arrhythmias in dialysis patients is linked not only to the high prevalence of structural heart disease but also to underlying perturbations in electrophysiologic properties. Coronary disease, $\mathrm{LVH}$, and systolic dysfunction are known for their strong remodeling effects of cardiac ion channels resulting in the acquired cardiac channelopathies that can prolong ventricular repolarization [14]. Such an adverse modulation of the cardiac electrophysiological matrix is characterized by a progressive reduction of the naturally redundant $\mathrm{K}^{+}$ channels and concomitant increase in the sensitivity to inhibition of the remaining $\mathrm{K}^{+}$channels. This results in further prolongation of repolarization and an elevation in the risk for lethal arrhythmias. In addition to the inherent alterations in ion channel modulation, electrolyte disturbances and exposure to multiple proarrhythmic medications play important roles in SCA in these patients.

Prevention of Sudden Cardiac Death in Dialysis Patients
Dialysis patients also demonstrate a withdrawal in parasympathetic tone and an increase in the sympathetic input to the heart. Alterations in heart rate variability (HRV) occur more frequently in dialysis patients and correspond with increased incidences of SCA and overall cardiovascular mortality [15]. Another measure, Twave alternans (TWA), reflects spatiotemporal heterogeneity of repolarization and serves as both a marker and a cause of malignant arrhythmias. In a study of TWA in chronic HD patients, $85 \%$ were found to have abnormal measures while a separate study found a twofold increase in the prevalence of abnormal TWA compared to controls $[16,17]$. Heart rate turbulence (HRT) measures cardiac vagal responsiveness and is an important predictor of cardiac events in heart failure patients. In dialysis patients, abnormal HRT was present in 57\%, though the predictive value for SCA has not been assessed [17].

\section{Dialysis-Related Risk Factors}

\section{Type of Dialysis: Peritoneal versus Hemodialysis}

Cardiovascular death rates and sudden cardiac death rates are similar between patients treated with peritoneal and hemodialysis. In a 5-year study of peritoneal dialysis patients, $24 \%$ of all deaths were due to SCA, a number strikingly similar to that observed in hemodialysis patients [13]. There is an interplay, however, between the type of renal replacement therapy, dialysis vintage, and risk of SCA with the relative hazard of cardiac arrest in hemodialysis compared to peritoneal dialysis varying with time after initiation of renal replacement therapy. The rate of cardiac arrest is about $50 \%$ higher in hemodialysis patients within the first 3 months of dialysis initiation, but they are similar at 2 years [18].

\section{Dialysate}

Rapid shifts in electrolyte concentrations can alter the action potential and support proarrhythmia. The use of low potassium dialysate appears to be a risk factor for SCA [19]. In a case-control study of 43,200 hemodialysis patients, the use of low potassium $(<2.0 \mathrm{mEq} / \mathrm{l})$ baths was associated with higher SCA risk, a finding that could not be explained by the pre-dialysis serum potassium levels. In a separate study comparing 400 reported cardiac arrest cases occurring in dialysis units against a nationally representative cohort of 77,000 hemodialysis patients, those that suffered an arrest were nearly twice as likely to have been dialyzed against a $0-$ or $1.0-\mathrm{mEq} / \mathrm{l}$ potassium 
Fig. 2. All-cause survival following implantation of ICDs/CRT-Ds, by modality, 1999-2008 [18].

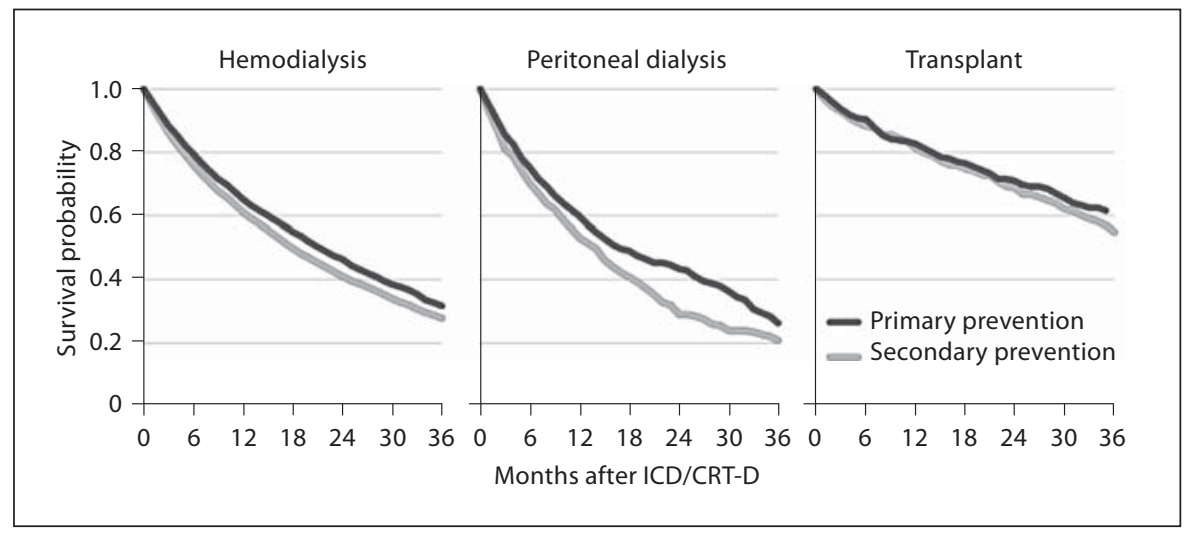

dialysate [20]. These suggest that avoiding low potassium dialysate prescriptions when possible may reduce the risk of SCA.

\section{Dialytic Intervals}

The incidence of SCA in hemodialysis patients is heterogeneously dispersed among dialytic intervals with the highest incidence observed during the 2-day interval prior to the first dialysis session of the week [12]. In a study of 32,065 patients, the rate of all-cause mortality, mortality from cardiac causes, mortality from cardiac arrest, mortality from myocardial infarction, and admissions for myocardial infarction, heart failure, stroke, and dysrhythmia were all higher on the day after the longest dialytic interval [21]. While it may be assumed that serum potassium plays a role, no such discrepancies were found in those patients with and without SCA [12]. Additionally, no differences in HRV, TWA, or HRT were noted between dialytic intervals [17]. Whether daily dialysis can reduce cardiovascular mortality and sudden death risk has yet to be determined.

\section{Role of Hyperphosphatemia}

In conjunction with hypocalcemia and 1,25-vitamin D deficiency, hyperphosphatemia can provoke smooth muscle proliferation, vascular calcification and coronary artherosclerosis $[5,6]$. Data from two national random samples of hemodialysis patients including 12,833 followed for 2 years showed that hyperphosphatemia, elevated parathyroid hormone levels, and elevations in calcium-phosphate $\left(\mathrm{Ca}-\mathrm{PO}_{4}\right)$ product were independently associated with death due to CAD and SCA [22]. While the cause of this link is speculative, the data suggest a role for elevated serum $\mathrm{PO}_{4}$ either in the development, progression, or the rupture of atheromatous plaques in the coronary arteries. Additionally, myocardial fibrosis induced by elevated levels of parathyroid hormone in patients with uremic cardiomyopathy may contribute to arrhythmogenesis, while hyperphosphatemia and Ca- $\mathrm{PO}_{4}$ product are thought to result in myocardial calcification with disruption of the structure and function of the cardiac conduction system [23].

\section{Prevention of Sudden Cardiac Arrest}

\section{Medical Therapies}

There is little evidence to date that any specific medical therapy has major impact on the prevention of SCA in the dialysis patient. $\beta$-Adrenergic blockers, a mainstay of therapy in patients with cardiovascular disease, has not been adequately evaluated in the dialysis population. In one small study of dialysis patients with dilated cardiomyopathy, the use of carvedilol was associated with a decrease in overall mortality and a non-significant reduction in SCA risk [24]. A report from the Dialysis Outcomes and Practice Patterns Study found that those patient receiving $\beta$-adrenergic blockers had lower overall mortality [25]. Retrospective studies have suggested a survival benefit from the use of angiotensin-converting enzyme inhibitors in dialysis patients, though a randomized trial of fosinopril failed to show any reduction in cardiovascular events including cardiac arrest [26]. Similar results were observed in the $4 \mathrm{D}$ trial which evaluated the efficacy of atorvastatin in type 2 diabetic dialysis patients. The study showed a non-significant $8 \%$ reduction in the composite endpoint of death from cardiac causes, fatal stroke, non-fatal myocardial infarction, or non-fatal stroke [7]. In a trial of an angiotensin II receptor blocker the results were more encouraging with those assigned to 
candesartan experiencing a reduction in cardiovascular events, total mortality and sudden deaths compared to the control arm [27]. Confirmatory studies are necessary before the routine use of angiotensin receptor blockers in dialysis patients without heart failure can be recommended.

\section{Implantable Defibrillators for Sudden Death \\ Prevention}

While it may be tempting to assume that a population at high risk for SCA such as those on dialysis would naturally benefit from implantable cardiac defibrillators (ICD) therapy, this is not clearly the case. The competing risk of death from other causes and the elevated complication rate of ICD implantation appear to limit the utility of these devices in dialysis patients.

Unlike in the general population, there are no randomized studies that have evaluated ICDs in dialysis patients who have survived SCA. Retrospective evidence suggests that ICDs may marginally lower short-term mortality. Data on 2,232 dialysis patients who received an ICD for secondary prevention were compared to 8,928 controls. There was a modest but significant reduction in mortality in years 1 and 2, but a convergence in survival rates at 3 years [28]. The USRDS reports median survival of only 18 months in dialysis patients receiving ICDs regardless of indication and well below that of non-dialysis ICD recipients (fig. 2) [18]. Nonetheless, current ICD guidelines do not distinguish between patients with and without CKD and in the absence of controlled trial data it appears that device implantation for secondary indications is reasonable.

For primary prevention of SCA, the interaction between CKD and ICD benefit is also poorly delineated. The benefit of the ICD for primary prevention of SCA appears to be attenuated in CKD patients in general with every 10 -unit decrease in estimated glomerular filtration rate associated with a $17 \%$ increase in SCA risk even with the device in place [29]. As with secondary prevention, there are no clinical trial data to guide ICD implantation for primary prevention in the dialysis population.

There are several reasons why ICDs may not prolong the life of the dialysis patient. First, defibrillation thresholds are known to be elevated in this population, raising the possibility that the device may be unable to terminate VT/VF should it occur. Second, complications including infections, bleeding, and lead dislodgement are all higher in the dialysis cohort as is compromise of vascular access [30]. Similarly, perioperative mortality is increased fivefold among dialysis patients undergoing ICD implantation [31]. Third, it is also possible that arrhythmic events in this population may simply be an epiphenomenon of impending cardiovascular collapse. Therefore, any device that simply terminates life-threatening arrhythmias may have little impact on long-term mortality. This is supported by a comparison of dialysis and non-dialysis ICD recipients that showed that while dialysis is the strongest predictor of appropriate ICD therapy for VT/ $\mathrm{VF}$, it is also the strongest predictor of mortality [32].

\section{Future Directions}

The growing prevalence of dialysis and its attendant risk of SCA makes risk stratification and prevention important goals. Unfortunately, the failure to include large numbers of dialysis patients in most major cardiology trials has limited our ability to better care for these individuals. Disease-specific, large-scale prospective cohort studies are required to define risk factors for SCA. Barriers should be removed to allow data linkage between administrative databases, registries, and clinical trial data. Randomized trials are needed to assess a wide spectrum of interventions as basic as $\beta$-adrenergic blockers and as complicated as ICDs (possibly subcutaneous), sympathetic denervation, and changes in dialysis itself.
References

\footnotetext{
Pun PH, Smarz TR, Honeycutt EF, Shaw LK, Al-Khatib SM, Middleton JP: Chronic kidney disease is associated with increased risk of sudden cardiac death among patients with coronary artery disease. Kidney Int 2009;76: 652-658.

2 Eknoyan G, Beck GJ, Cheung AK, et al: Effect of dialysis dose and membrane flux in maintenance hemodialysis. N Engl J Med 2002;347:2010-2019.

3 Lafrance JP, Nolin L, Senecal L, Leblanc M: Predictors and outcome of cardiopulmonary resuscitation calls in a large haemodialysis unit over a seven-year period. Nephrol Dial Transplant 2006;21:1006-1012.

-4 Takeda K, Harada A, Okuda S, et al: Sudden death in chronic dialysis patients. Nephrol Dial Transplant 1997;12:952-955.

5 Herzog CA, Mangrum JM, Passman R: Sudden cardiac death and dialysis patients. Semin Dial 2008;21:300-307.
} 
6 Reddan DN, Szczech LA, Tuttle RH, et al: Chronic kidney disease, mortality, and treatment strategies among patients with clinically significant coronary artery disease. J Am Soc Nephrol 2003;14:2373-2380.

7 Wanner C, Krane V, Marz W, et al: Atorvastatin in patients with type 2 diabetes mellitus undergoing hemodialysis. N Engl J Med 2005;353:238-248.

-8 Herzog CA, Strief JW, Collins AJ, Gilbertson DT: Cause-specific mortality of dialysis patients after coronary revascularization: why don't dialysis patients have better survival after coronary intervention? Nephrol Dial Transplant 2008;23:2629-2633.

9 Foley RN, Parfrey PS, Sarnak MJ: Clinical epidemiology of cardiovascular disease in chronic renal disease. Am J Kidney Dis 1998; 32:S112-S119.

10 Mark PB, Johnston N, Groenning BA, et al: Redefinition of uremic cardiomyopathy by contrast-enhanced cardiac magnetic resonance imaging. Kidney Int 2006;69:18391845.

$\checkmark 11$ Ayus JC, Mizani MR, Achinger SG, Thadhani R, Go AS, Lee S: Effects of short daily versus conventional hemodialysis on left ventricular hypertrophy and inflammatory markers: a prospective, controlled study. J Am Soc Nephrol 2005;16:2778-2788.

$\checkmark 12$ Bleyer AJ, Hartman J, Brannon PC, ReevesDaniel A, Satko SG, Russell G: Characteristics of sudden death in hemodialysis patients. Kidney Int 2006;69:2268-2273.

13 Wang AY, Lam CW, Chan IH, Wang M, Lui SF, Sanderson JE: Sudden cardiac death in end-stage renal disease patients: a 5-year prospective analysis. Hypertension 52010;6: 210-216.

14 Tomaselli GF, Marban E: Electrophysiological remodeling in hypertrophy and heart failure. Cardiovasc Res 1999;42:270-283.
15 Oikawa K, Ishihara R, Maeda T, et al: Prognostic value of heart rate variability in patients with renal failure on hemodialysis. Int J Cardiol 2009;131:370-377.

-16 Patel RK, Mark PB, Halliday C, et al: Microvolt $\mathrm{T}$-wave alternans in end-stage renal disease patients - associations with uremic cardiomyopathy. Clin J Am Soc Nephrol 2011;6: 519-527.

17 Secemsky EA, Verrier RL, Cooke G, et al: High prevalence of cardiac autonomic dys function and T-wave alternans in dialysis patients. Heart Rhythm 2011;8:592-598.

18 US Renal Data System 2006 Annual Data Report: Atlas of End-Stage Renal Disease in the United States. Bethesda, NIH, National Institute of Diabetes and Digestive and Kidney Diseases, 2010.

19 Pun PH, Lehrich RW, Honeycutt EF, Herzog CA, Middleton JP: Modifiable risk factors associated with sudden cardiac arrest within hemodialysis clinics. Kidney Int 2011;79: 218-227.

20 Karnik JA, Young BS, Lew NL, et al: Cardiac arrest and sudden death in dialysis units. Kidney Int 2001;60:350-357.

21 Foley RN, Gilbertson DT, Murray T, Collins AJ: Long interdialytic interval and mortality among patients receiving hemodialysis. $\mathrm{N}$ Engl J Med 2011;365:1099-1107.

22 Ganesh SK, Stack AG, Levin NW, HulbertShearon T, Port FK: Association of elevated serum $\mathrm{PO}_{4}, \mathrm{Ca} \times \mathrm{PO}_{4}$ product, and parathyroid hormone with cardiac mortality risk in chronic hemodialysis patients. J Am Soc Nephrol 2001;12:2131-2138.

23 Block GA, Hulbert-Shearon TE, Levin NW, Port FK: Association of serum phosphorus and calcium $\times$ phosphate product with mortality risk in chronic hemodialysis patients: a national study. Am J Kidney Dis 1998;31:607-617.

24 Cice G, Ferrara L, D’Andrea A, et al: Carvedilol increases two-year survivalin dialysis patients with dilated cardiomyopathy: a prospective, placebo-controlled trial. J Am Coll Cardiol 2003;41:1438-1444.
25 Ritz E, Dikow R, Adamzcak M, Zeier M: Congestive heart failure due to systolic dysfunction: the Cinderella of cardiovascular management in dialysis patients. Semin Dial 2002;15:135-140.

26 Zannad F, Kessler M, Lehert P, et al: Prevention of cardiovascular events in end-stage renal disease: results of a randomized trial of fosinopril and implications for future studies. Kidney Int 2006;70:1318-1324.

27 Takahashi A, Takase H, Toriyama T, et al: Candesartan, an angiotensin II type-1 receptor blocker, reduces cardiovascular events in patients on chronic haemodialysis - a randomized study. Nephrol Dial Transplant 2006;21:2507-2512.

28 Charytan DM, Patrick AR, Liu J, et al: Trends in the use and outcomes of implantable cardioverter-defibrillators in patients undergoing dialysis in the United States. Am J Kidney Dis 2011;58:409-417.

29 Goldenberg I, Moss AJ, McNitt S, et al: Relations among renal function, risk of sudden cardiac death, and benefit of the implanted cardiac defibrillator in patients with ischemic left ventricular dysfunction. Am J Cardiol 2006;98:485-490.

30 Dasgupta A, Montalvo J, Medendorp S, et al: Increased complication rates of cardiac rhythm management devices in ESRD patients. Am J Kidney Dis 2007;49:656-663.

31 Aggarwal A, Wang Y, Rumsfeld JS, Curtis JP, Heidenreich PA: Clinical characteristics and in-hospital outcome of patients with endstage renal disease on dialysis referred for implantable cardioverter-defibrillator implantation. Heart Rhythm 2009;6:15651571.

32 Robin J, Weinberg K, Tiongson J, et al: Renal dialysis as a risk factor for appropriate therapies and mortality in implantable cardioverter-defibrillator recipients. Heart Rhythm 20063:1196-1201. 